IJCOM 2021 November;1(2):44-55

\title{
Impact of COVID-19 Pandemic on Paediatric Trainees in Belgium
}

\author{
Tine Alliet ${ }^{1,2}$, Thomas Gestels ${ }^{1,3}$, Siel Daelemans ${ }^{1,4}$, Levi Hoste ${ }^{1,5,6^{*}}$ \\ ${ }^{1}$ Junior Representation of the Flemish Society for Paediatrics (Jong VVK) \\ ${ }^{2}$ Department of Paediatrics, University Hospital of Leuven, Leuven, Belgium \\ ${ }^{3}$ Department of Paediatrics, Antwerp University Hospital, Edegem, Belgium \\ ${ }^{4}$ Department of Paediatrics, University Hospital of Brussels, Brussels, Belgium \\ ${ }^{5}$ Faculty of Medicine and Health Sciences, Ghent University, Ghent, Belgium \\ ${ }^{6}$ Department of Paediatric Immunology and Pulmonology, Center for Primary Immunodeficiency Ghent, Jeffrey Modell Diagnosis and \\ Research Centre, Ghent University Hospital, Gent, Belgium \\ *Coresponding address: Levi Hoste \\ Email: levi.hoste@ugent.be
}

\begin{abstract}
Background and objectives: The COVID-19 pandemic has put strain on the activities and well-being of health care workers. We aimed to measure the direct and indirect impact on a personal and professional level for paediatric trainees in Flanders, Belgium.

Study design: Junior representatives of the Flemish Society for Paediatrics (Jong VVK) conducted a longitudinal study among their fellow paediatric trainees. The impact of COVID-19 on daily tasks, education and emotional well-being for the first (March-April 2020) and second wave (October-November 2020) of the pandemic were studied.

Results: One hundred and nineteen surveys were completed in the first wave, representing data of 51\% $(119 / 233)$ of the total number of paediatric trainees in Flanders. Eighty surveys were completed in the second wave. Educational program changes occurred in $25 \%$ $(30 / 119)$ of trainees and more than half $(61 \% ; 72 / 119)$ described the pandemic as an impediment for their educational progress. The perception of impaired education persisted for $30 \%$ of the responders $(24 / 80)$ during the second wave. One out of three $(30 \% ; 35 / 119)$ felt their job was more exhausting than usual and 38\% (45/119) perceived more stress at work. These numbers were comparable at both time points. Increases in stress paralleled with increased irritability in daily life and poorer sleep quality.

Conclusions: COVID-19 had an important impact on the daily tasks, education and emotional well-being of the paediatric trainees. Medical training centres should be aware that there is a perception of impediment on the educational program of the paediatric trainee. Keywords: COVID-19, residency, trainees, paediatrics
\end{abstract}




\section{Introduction}

The novel coronavirus, known as severe acute respiratory syndrome coronavirus 2 (SARS-CoV-2) and causing coronavirus disease 2019 (COVID-19), has spread rapidly from a local cluster of severe pneumonia cases in Wuhan (China) in December 2019 to a pandemic crisis. It has led to a massive disruption of individual and societal structures and caused important health care-associated and socioeconomic consequences in all parts of the world in a few weeks' time. ${ }^{1}$ Entire health care systems were forced to swiftly adapt to this unprecedented scenario to prepare for a massive influx of COVID-19-associated hospital admissions and to prevent collateral damage in patients with alternative or chronic conditions.

During the pandemic, Belgium recorded the highest death rate in the world as a consequence of COVID-19 in March 2020 (84.45 deaths per 100,000 population) and with a rate of 1,735 cases per 100,000 inhabitants in 14 days, it had the highest rate of SARS-CoV-2 infections in Europe in November 2020. ${ }^{2}$

The unprecedented measures that were necessarily taken inside and outside the hospitals have put strain on the personal and professional activities and wellbeing of health care workers. ${ }^{3}$ Although COVID-19 mainly presents with (severe) disease in the elderly population with co-morbidities and therefore primarily affects adult care, other specialties were not spared from the consequences of this crisis either. Of all health care work forces, especially trainees in several medical specialties were exposed to COVID-19 patient care and felt an immediate or indirect impact from the crisis. ${ }^{4}$ This observation has been made by various medical specialties such as cardiology, urology, plastic surgery, neurosurgery, radiology, otolaryngology, ophthalmology, anaesthesiology, cardiothoracic surgery and general surgeons. ${ }^{5-15}$ COVID-19 also had an impact on the curriculum of (medical) students which was yet described in detail. ${ }^{16}$

Medical trainees are a unique group of doctors who are still in a learning process to become a specialist but who, simultaneously, are also responsible for the care of patients. Paediatrics is a specialty that is familiar with infectious diseases and is acquainted to tackle epidemics causing temporary periods of high clinical demands (e.g., respiratory infections in winter months). Nevertheless, it is unlikely that the unseen measures necessary to tackle the challenges associated with the COVID-19 pandemic and the inevitable impact on health care systems, work forces and (lack of adequate) resources would not put significant burden on trainees in paediatrics. Few articles were published on the experiences of paediatric trainees during COVID-19. Sanghavi et al. found that $21 \%$ of American paediatric trainees displayed symptoms of a mild depression and $7 \%$ of a moderate depression. ${ }^{17}$ Babal et al. found anxiousness in $71 \%$ of paediatric trainees in the USA, angriness in 53\%, sadness in 53\%, and detachment in $41 \% .^{18}$

In this study, we aimed to measure the direct and indirect impact of the COVID-19 crisis on a personal and professional level in Flemish paediatric trainees.

\section{Materials and Methods}

An electronic questionnaire was drafted by junior representatives of the Flemish Society for Paediatrics (Jong VVK) and was tested for its technical functionality prior to dissemination. The questionnaire was sent out to fellow trainees in paediatrics at the four Flemish universities (University of Antwerp, Ghent University, Catholic University of Leuven and Free University of Brussels), either by contacting them directly or by e-mail. In the questionnaire, participants were informed about the duration of the survey, the investigators and the purpose of the study. To minimize recall bias, data concerned current opinions and the participants were asked to recollect data on the past two months. Two similar surveys were sent out after each epidemic wave in Belgium: the first at the end of April and the second at the end of November. Trainees that initiated their training in October 2020 were excluded in the second survey because they were not able to answer questions that compared the first and the second wave. Trainees were given a deadline of two weeks to complete the survey. No incentives were offered. The number of questions per page was two to five with a total of seven pages. All questions had to be answered before the questionnaire could be sent to the investigators. The survey consisted of basic demographic data (such as the university training hospital, current hospital and years of training) and three parts regarding the pandemic. The first part mainly addressed changes in daily tasks in the hospital (e.g., care for adult patients, working in other departments, changes in working hours or 
working from home), while the second and third part explored the impact on an educational level (added value or obstruction as a result of the crisis) and psychosocial well-being (e.g., emotional stress, fatigue, irritability), respectively. In this final part, trainees were asked to contemplate on 16 different statements and relate to them by answering on a Likert scale (completely agree - rather agree - neutral - rather disagree - completely disagree). The second survey contained multiple comparing questions about differences between the first and second peak. Answers were anonymized for the researchers. Junior trainees were defined as first- or second years. Senior trainees were defined as being at least in their third year of education (standard trajectory in Belgium comprises of five years of fulltime education).

Non-parametric statistical tests were used, according to results of normality tests (Shapiro-Wilk). MannWhitney $U$ and Kruskal Wallis test were used in the data analysis depending on the number of variables. For statistical analysis, dichotomous variables were created from statements declaring agreement (completely/rather agree $=$ true , neutral $/$ rather $/$ completely disagree $=$ false .

\section{Results}

One hundred and nineteen surveys were completed by individual trainees after the first epidemic wave, which consists of $51 \%(119 / 233)$ of the total number of paediatricians currently in training at the four targeted universities (Figure 1). The second questionnaire was filled in by 80 trainees, a lower number that could mainly be attributed to exclusion of the first years in training. Most of the trainees were part of a clinical training program. Only 19 out of the $119(16 \%)$ trainees were involved in a research program. This fraction was slightly higher in the second survey $(15 / 80 ; 18,8 \%)$. There was a homogeneous spread of years in training (seniority), with an expected overrepresentation of second years in the first survey and third years in the second survey, as this year consisted of an almost double cohort due to shortening of the medicine study program from seven years to six years (resulting in two cohorts of medical students graduating at the same time). As stated above, first-year trainees were excluded in the second survey. The majority $(55 \% ; 65 / 119$ and $59 \%$; 47/80) of respondents was working in a university hospital at the

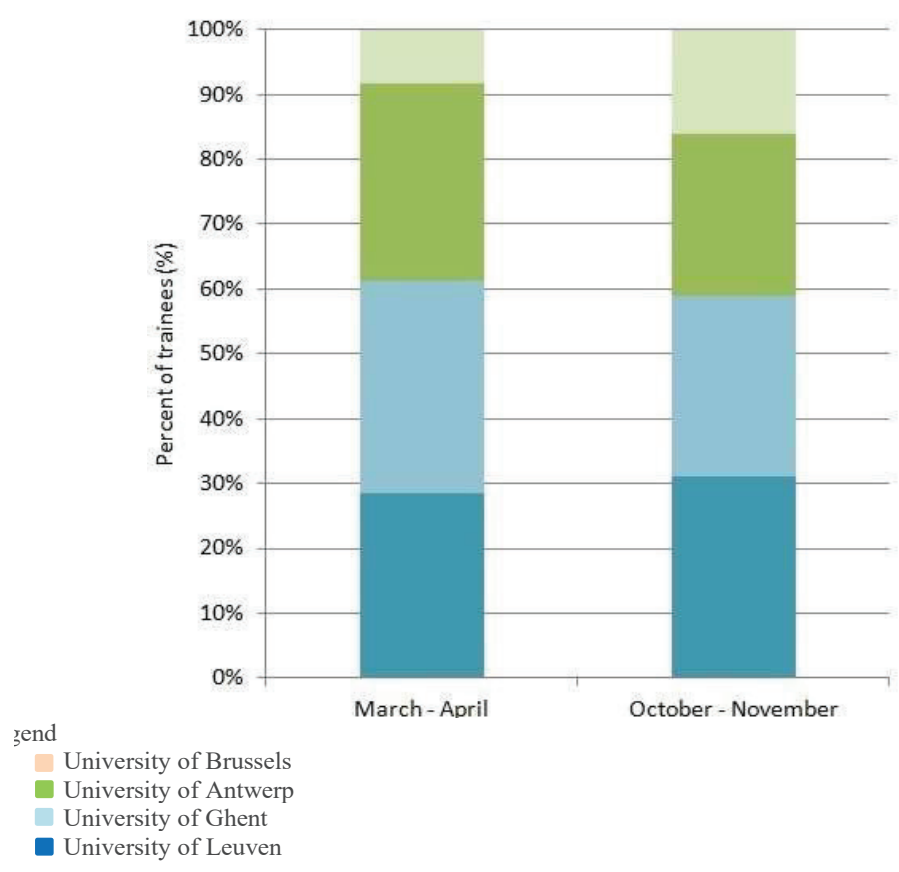

Figure 1. Response per university for both questionnaires

All four Flemish universities were represented in both questionnaires (March-April and October-November). 


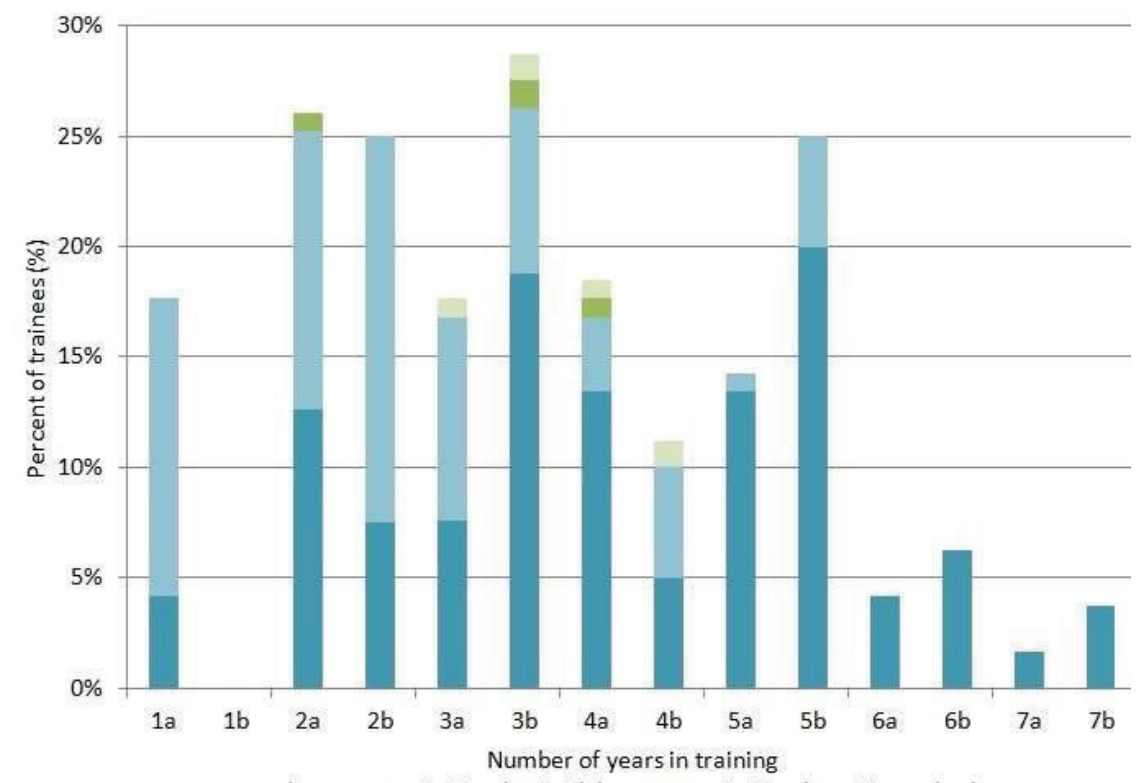

(a: response in March - April, b: response in October - November)

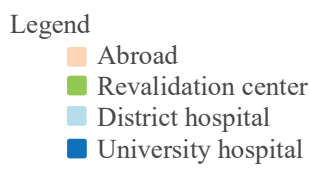

Figure 2. Working environment

This figure shows the percentage of trainees working in a university hospital, a district hospital, a revalidation center or abroad for each training year in the first questionnaire (March-April $=\mathrm{a}$ ) and second questionnaire (October-November $=\mathrm{b}$ ).

time the questionnaire was sent out. The second largest group (39\%; 47/119 and 35\%; 28/80) was working in a district hospital. The remaining seven trainees from the first survey were either employed in a research facility (2), a revalidation centre (2), abroad (2) or doing a combination of on calls and working from home (1). In the second survey the remaining responders were either working abroad (2), in a revalidation centre (1) or in a research facility (2) (Figure 2). Two thirds (63\%; 50/80) were working in different hospitals during the two epidemic waves.

While most of the paediatric training programs were not directly affected by the COVID-19 pandemic, both during the first and second peak, 1 out of 4 respondents did experience a change in their program. Trainees working in a university centre in the first lockdown (23/65) were significantly more affected compared to trainees in district hospitals $(7 / 47 ; \mathrm{P}<0.05)$. For most of the trainees with a change in program $(60 \% ; 18 / 30$ in the first peak and 57\%; 12/21 in the second peak), the adjustments of their schedule were implemented for less than or equal to one month. Nevertheless, for the remaining $40 \%(12 / 30)$ and $42 \%(9 / 21)$, more than 1 month of their training was affected.

When analysing the workload and working hours of the first wave, most trainees experienced either no difference or a decrease in both workload and working hours compared to their usual workload and working hours (Figure 3). Only 13\% (16/119) felt their workload had increased and 12\% (15/119) replied that they had worked more hours. However, an increase in workload was significantly associated with increased working hours $(\mathrm{P}<0.001)$, feelings of unsafety $(\mathrm{P}<0.001)$, more stress and exhaustion at work $(\mathrm{P}<0.05)$, needing to do uncomfortable tasks $(\mathrm{P}<0.05)$ and worse sleep quality $(\mathrm{P}<0.05)$. This was unchanged for the majority of 


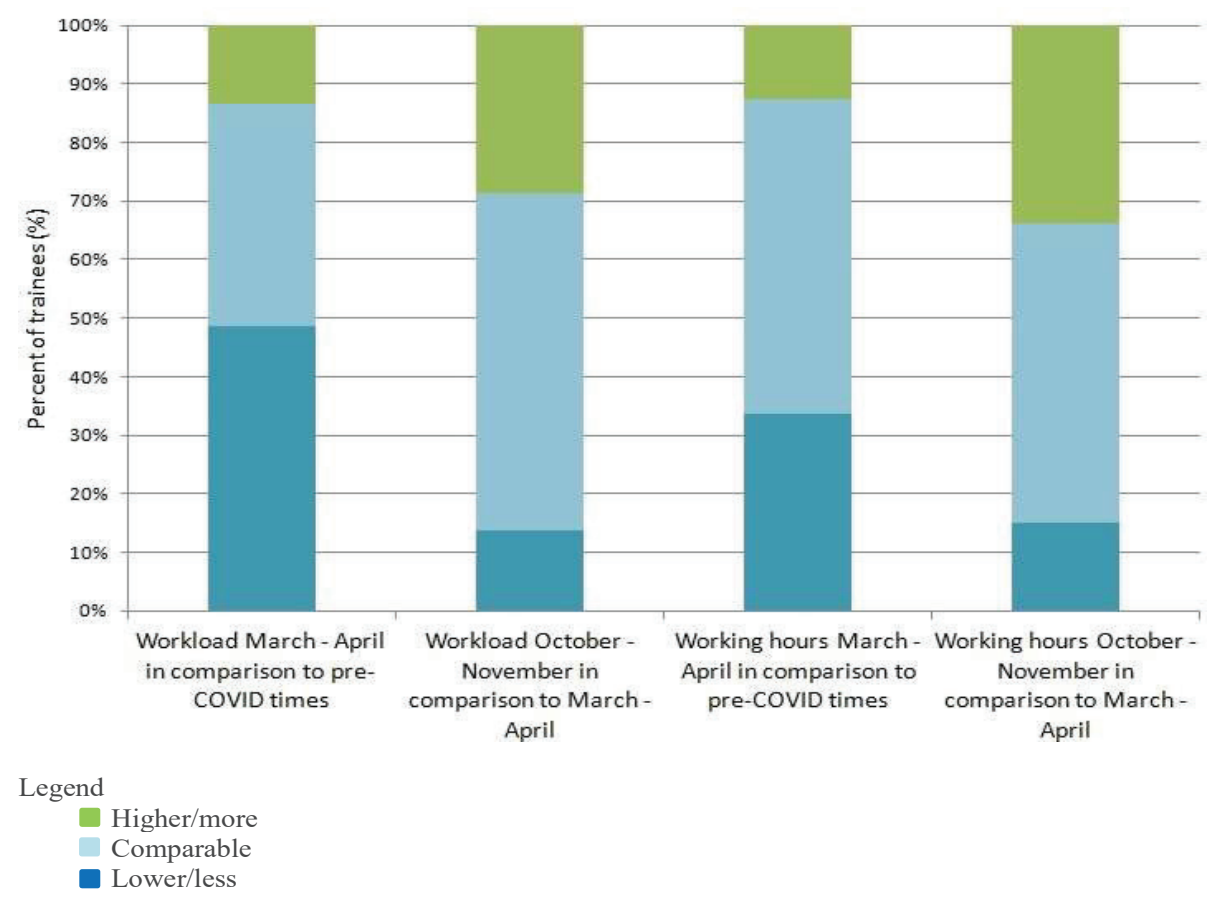

Figure 3. Workload and working hours

This figure showed the perceived workload (left) and working hours (right) from the first wave (March-April) compared to pre-COVID-times and the second wave (October-November) compared to the first wave.

trainees during the second wave, although 29\% (23/80) felt their workload had increased when compared to the first wave and 34\% (27/80) worked more hours as compared to the first wave (Figure 3).

In contrast to workload and hours, the content of the working day did change for most paediatric trainees during the first peak. Only a third of respondents (36\%; 43/119) said their daily job had not changed at all. Often this meant doing different tasks within their own department. Only $8 \%(9 / 119)$ were asked to help out on the adult wards and 5\% (6/119) were assigned a different function (e.g., nursing care) (Figure 4). Senior trainees were significantly more at risk for having a change in their program $(25 / 67 ; \mathrm{P}<0.05)$ as compared to juniors (7/52). On the other hand, juniors were significantly more at risk of carrying out another function in the hospital $(6 / 52 ; \mathrm{P}<0.05)$, whereas no single senior trainee carried out a non-medical task during the crisis.
During the second COVID-19 wave, working day content was unaffected for $74 \%$ (59/80) of the trainees. In contrast to the first wave, only 5\% (4/80) were transferred to assist in patient care on adult units (Figure 4).

Remarkable is that almost half of all the trainees who answered the first questionnaire (44\%; 52/119) were at least partially working from home (Figure 4), which occurred significantly more in trainees working in university hospitals (45/65) as compared to district centres (3/47; $\mathrm{P}<0.001)$. Since only $14 \%$ (17/119) of participants had to self-isolate due to symptoms, this was not the main reason for the increase in homework. Working from home was most likely initiated to prevent all medical staff to be present at the same time, to ensure physical distancing and thereby preventing the spread of the virus. The proportion of trainees working from home did not declare being more stressed at home, did 


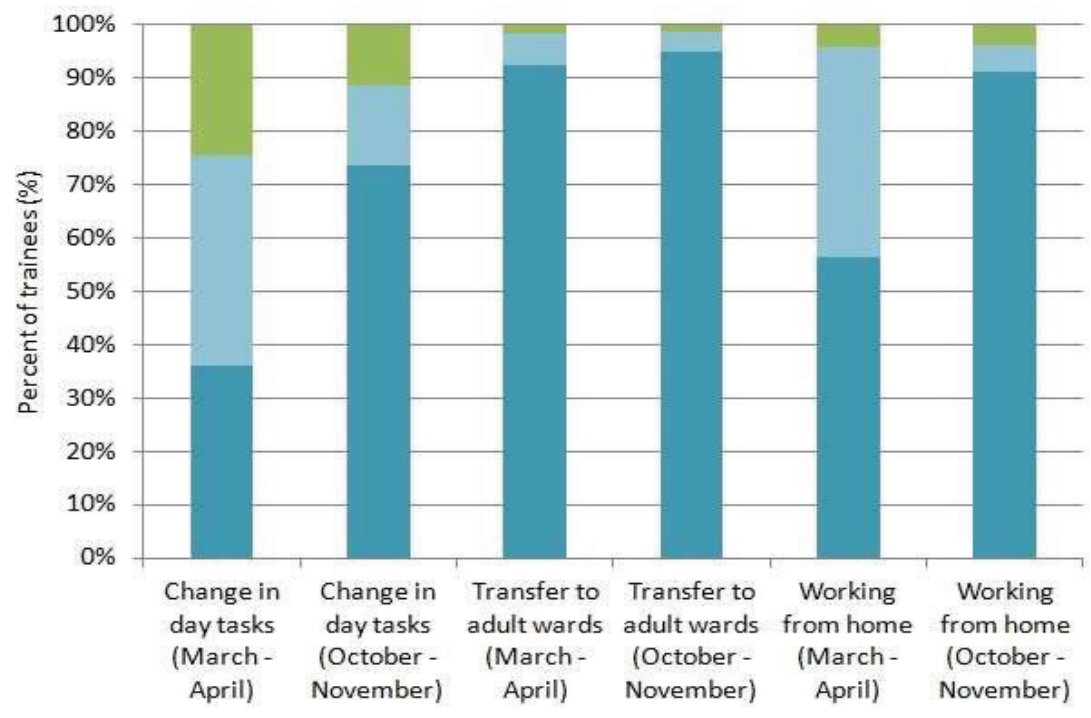

Legend

Yes, more than $50 \%$ of the time

Yes, but less than $50 \%$ of the time

No

Figure 4. Change in content of the working day

Proportion of trainees who experienced a change in the day tasks (left), who were transferred to an adult ward (middle) and who were working from home (right) in the first and second questionnaire.

not feel less part of a team or did not feel useless in the crisis. The number of trainees working from home decreased massively in the second wave $(9 \% ; 7 / 80)$ (Figure 4). Absence because of health issues due to confirmed or suspected COVID-19 remained rather exceptional $(5 \% ; 4 / 80)$.

Even though some of the training schedules had fewer working hours compared to the regular working rota, income was unchanged for the large majority $(89 \% ; 106 / 119)$ of the trainees. For 3 of the 119 responders pay had increased, while 10/119 stated to have lost income. A single trainee even reported an income reduction of more than $25 \%$. Working from home did not result in a higher proportion of trainees with a reduction in income. Juniors however, had a more frequent decrease in payment during the crisis $(9 / 52)$ as compared to seniors $(1 / 67 ; \mathrm{P}<0.05)$.

Half of the trainees $(56 \% ; 67 / 119)$ declared having a great interest in paediatric specialties directly related to COVID-19 (respiratory diseases, infectious diseases, epidemiology, immunology, acute paediatrics or intensive care). Only 16\% (19/119) showed little interest. Most trainees stated that the pandemic did not change their interest level in these domains. More interest in COVID-19 related specialties did not significantly increase well-being. The pandemic did not influence the choice for paediatrics for the vast majority (71\%; 85/119). For 28\% (33/119) their career choice was even positively reinforced.

Unfortunately, 61\% (72/119) of paediatric trainees experienced this pandemic as an impediment for their educational progress. This remained the case for $70 \%$ $(56 / 80)$ in the second questionnaire, notwithstanding $64 \%$ (36/56) stated this had already improved when compared to the first peak.

Despite the fact that a third of the trainees (34\%; 40/119) were provided with COVID-19 related courses by their training institute and about 29\% (34/119) were able to enjoy COVID-19 related teaching in their training centre, $81 \%(96 / 119)$ felt they mainly had to educate themselves to expand their knowledge about 


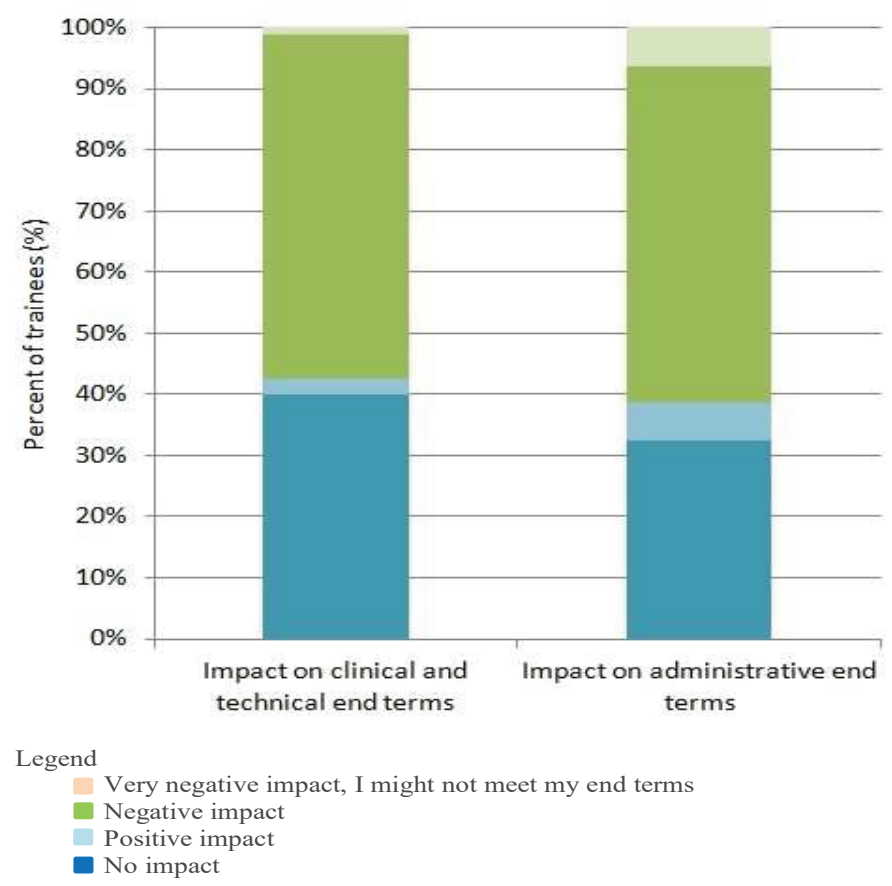

Figure 5. Impact on end terms

Trainees' perception of the impact of the COVID-19 crisis in meeting their clinical and technical (left) and administrative (right) end terms.

the new coronavirus. This remained unchanged in the second wave, and an even larger proportion stated to mainly have been responsible themselves for their education on COVID-19 (84\%; 67/80). Declaring that self-study comprised the largest part of education was found more frequently in trainees employed in district hospitals (42/47), as compared to trainees in university centres $(48 / 65 ; \mathrm{P}<0.05)$.

Education in general seems to have improved during this coronavirus pandemic when comparing the educational availabilities at the beginning of the pandemic to the situation by the end of the year, according to $41 \%(33 / 80)$ of the responders. Online teaching and re-introduction of teaching in the hospital were mentioned most often as an explanation for this improvement. Nevertheless, a relevant proportion of trainees did not experience this improvement. Twentynine percent $(23 / 80)$ of the trainees felt little to no change and $30 \%(24 / 80)$ even responded that education was even worse in the second wave when compared to the first one.
Trainees were asked to evaluate the effect of the entire pandemic on their clinical, technical and administrative end terms (Figure 5). Administrative end terms included master papers, other publications and professional recognition criteria. Concerning clinical and technical end terms, 40\% (32/80) felt no impact, 3\% (2/80) experienced a positive impact but the majority $(57 \% ; 46 / 80)$ felt a negative impact of which one trainee even stated that this might influence his/her end terms attainment. For the administrative end terms, an even greater proportion declared to have encountered a negative impact $(61 \% ; 49 / 80)$, of which five trainees feared failure to achieve their end terms.

As reported above, the number of working hours was not greatly affected for most trainees. Nonetheless, one out of three $(29 \% ; 35 / 119)$ felt their job was more exhausting than usual and 38\% (45/119) was more stressed at work (Figure 6). This observation was significantly more frequent in junior trainees $(25 / 52)$, as compared to seniors $(20 / 67 ; \mathrm{P}<0.05)$. These numbers remained largely unchanged in the second questionnaire. 


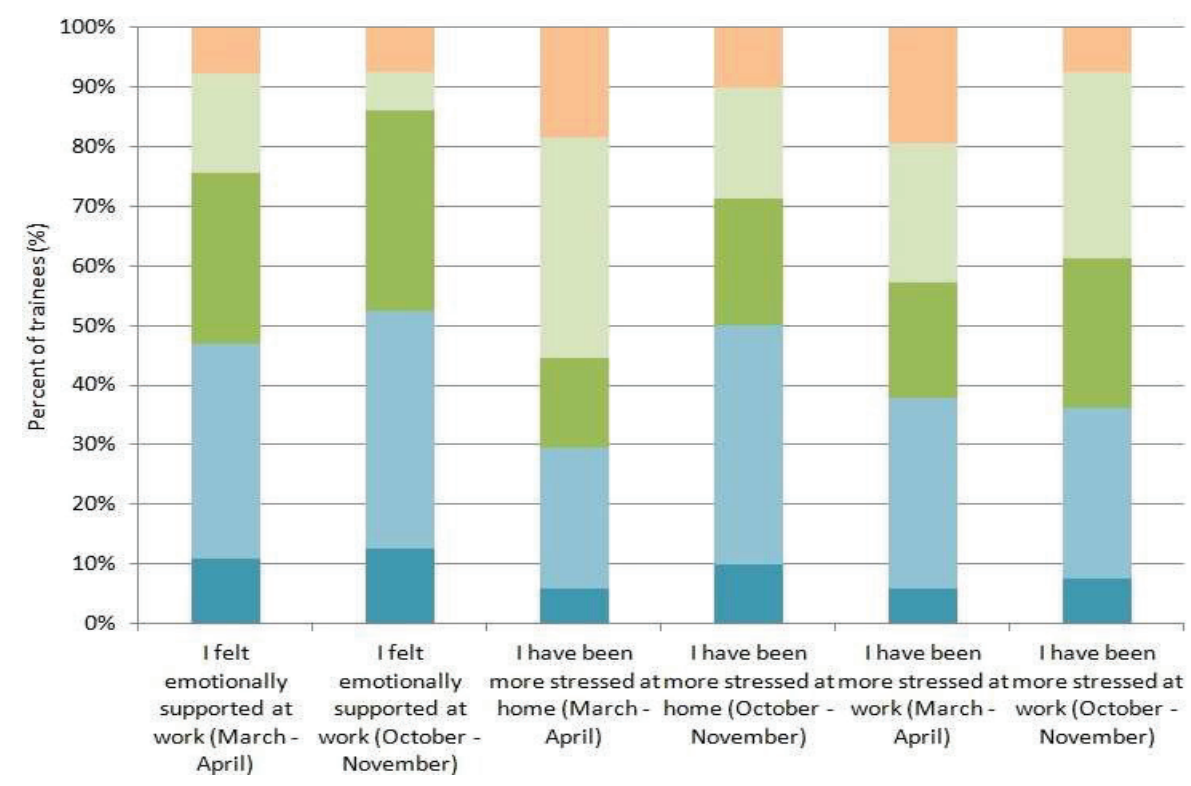

Legend

$$
\begin{aligned}
& \text { Completely disagree } \\
& \text { Rather disagree } \\
& \text { Neutral } \\
& \text { Rather agree } \\
& \text { Completely agree }
\end{aligned}
$$

Figure 6. Stress and emotional support

This figure shows how trainees felt they were supported at work (left) and if they experienced more stress at home (middle) or at work (right) in both waves

This increase in stress was often present at home too, as $43 \%(51 / 119)$ of the trainees stated their stress levels at home were higher than usual in the first wave with a slight increase in the second wave $(50 \% ; 40 / 80)$ (Figure 6). A smaller group of $21 \%$ (25/119) felt unsafe at work and had concerns for their own health. These concerns were troubling more trainees in the second wave $(32 \% ; 25 / 80)$. Trainees with feelings of unsafety declared significant higher workload $(13 / 35 ; \mathrm{P}<0.001)$ and working hours $(10 / 35 ; \mathrm{P}<0.05)$, more exhausting work $(26 / 35 ; \mathrm{P}<0.001)$, more stress at work $(26 / 35$; $\mathrm{P}<0.001)$ and worse sleep quality $(18 / 35 ; \mathrm{P}<0.05)$. The increase in stress resulted into increased irritability in daily life for $26 \%(31 / 119)$ and in poorer sleep quality for $29 \%(35 / 119)$ of the trainees which remained unchanged during the second peak.

Unfortunately, less than half of the trainees answered positively when asked about the provision of emotional support at work (47\%; 56/119) (Figure 6). A quarter $(24 \% ; 29 / 119)$ disagreed when asked whether they were sufficiently supported. Sadly, the availability of emotional support at work did not change during the second wave according to the majority of the responders (69\%; 55/80).

When asked whether the trainees felt more part of a team than usual during the pandemic, almost half of them $(45 \% ; 53 / 119)$ had no opinion about this subject. A third $(34 \% ; 41 / 119)$ agreed and unfortunately a fifth $(21 \% ; 25 / 119)$ disagreed. It is worth noting that feeling emotionally supported at work significantly increased the proportion of trainees declaring the crisis as an added value $(23 / 41 ; \mathrm{P}<0.05)$ as compared to not being supported $(24 / 78)$.

Exactly half of the trainees replied that relaxing outside of work has been more difficult than usual due to the lockdown restrictions. This number was significantly higher in the second wave $(88 \% ; 70 / 80)$. For another $14 \%(17 / 119)$ relaxation was more difficult due to work or other reasons (e.g., family related). Controversially, almost a quarter $(23 \% ; 27 / 119)$ stated that relaxing 
outside of work was easier than usual. Feelings of stress at work correlated with higher proportions of workload $(\mathrm{P}<0.05)$, needing to do uncomfortable tasks $(\mathrm{P}<0.05)$, feeling more stress at home $(\mathrm{P}<0.05)$, having worse sleep $(\mathrm{P}<0.05)$, feelings of exhaustion and unsafety $(\mathrm{P}<0.001)$, and feeling less part of a team $(\mathrm{P}<0.05)$ or less useful in the crisis $(\mathrm{P}<0.05)$. Even though we know paediatric patients were less severely affected by COVID-19, 15\% (18/119) declared that they were confronted more often with severe illness and end-of-life decisions during this pandemic. This number remained high in the second survey $(19 \% ; 15 / 80)$.

There has been a lot of media attention for medical staff, hospital funding and healthcare organisation due to the current pandemic. A third $(34 \% ; 40 / 119)$ of trainees however, felt their job should receive more respect after this pandemic. More than half of the respondents $(57 \%$; 68/119) declared they did not feel very useful in the battle against SARS-CoV-2 and only a minority $(19 \% ; 23 / 119)$ confirmed that they felt useful during the pandemic. Contradictory with these results, only $21 \%(25 / 119)$ wished they could have played a different role in the pandemic. These numbers were comparable in the second survey.

\section{Discussion}

This longitudinal study, conducted in paediatric trainees in Flanders, Belgium, confirms our research hypothesis that although the paediatric specialty was relatively spared from the direct consequences of the COVID-19 pandemic, its impact was indirectly felt in the daily tasks, education and emotional well-being of the trainees.

Although the majority of trainees in this study saw no reduction in workload or hours in the clinic, the overall appreciation of their education was insufficient and was frequently labelled as obstructive for their training goals. Strikingly, although still in a didactical environment, knowledge concerning COVID-19 was most often gained from self-study. Although the pandemic evidently forced us to swiftly adapt traditional educational activities to an unfamiliar and largely virtual learning environment, the technological tools and skills that we currently employ should from now on pave the way for accessible distance learning to provide sustained educational programs for every trainee.
Thankfully, several actions with the aim to optimize trainees' well-being and education have already been proposed, such as adequate access to personal protective equipment, sufficient support (at the level of both the trainee program and the institutions) and instituting telehealth education programs (6). As the current pandemic forces us to leave the more traditional teaching model of high case volumes and passive learning behind, training centres should embrace these circumstances to deploy resources towards these internet- and video-based learning, simulator training and more individualised educational models. The deployment of such smartlearning technologies in order to limit the impact of the COVID-19 on the learning curves of trainees has already been proposed in other specialty trainings, such as urology, gastroenterology and dermatology.,19,20 Practical tips for supporting competency-based medical education have already been published. ${ }^{21}$ If sufficiently offered, these new training methods and technologies may allow for fulfilment of important and individualized educational goals that may not have been met in the past.

Besides medical education, the impact on mental well-being may not be forgotten. In this study, one out of three paediatric trainees were more stressed at work, and this was especially so for junior trainees This increase in stress resulted into increased irritability in daily life and poorer sleep quality. A quarter of the trainees did not feel sufficiently supported at work and the majority of trainees stated that relaxing outside of work was more difficult. Luckily most trainees were, despite this, still positive about their career choice. But, knowing this world crisis is a marathon and not a sprint, a long-lasting combination of these factors can contribute to the development of burnout. Burnout prevalence is known to be high in trainees, also during this pandemic. ${ }^{22,23}$ It is thus important in such a crisis to install monitoring systems early on and offer psychological support to those in need. Dispatching trainees to perform patient care outside their clinical competencies, such as is reported in this study, is however a risk. ${ }^{24}$ Feelings of anxiousness and vulnerability have indeed been observed in trainees serving on the pandemic's front lines, which was confirmed by an important share of our cohort. ${ }^{25}$ Safety concerns in particular were believed to be an important asset to these feelings. A survey among local residents (263 participants) in China already showed that $52 \%$ of participants felt horrified and apprehensive due to the pandemic. An open learning environment 
and resilience training programs have been suggested to respond to these feelings of hopelessness and burnout. ${ }^{26}$

Generally speaking, the COVID-19 pandemic could and should be an important learning opportunity, especially on generic skills such as professionalism, quality and safety in care and tackling ethical dilemmas that have risen during this crisis. Mastering these tools is highly valuable to every paediatrician and should transcend the subspecialty divisions. As such, both trainees with interests directly related to COVID-19 patient care (infectious diseases, immunology, respiratory medicine), but also trainees without these specific interests should be in a position to benefit from this period, and subsequently none of them should label this crisis as an obstruction for their educational goals. Considering the findings of this survey, prompt and customized action is thus required to meet the presumed educational shortcomings. COVID-19 is one of the many infectious diseases of the past decades and will most certainly not be the last for this generation. These outbreaks will most likely become more common given increasing urbanization rates, the widespread accessibility of air travel, and worsening climate change. ${ }^{27}$ Therefore, it is of most importance to take appropriate lessons from this period and seize the opportunity to adapt and act relevant in the preparedness for the next crisis. Priority areas central to promoting and maintaining the well-being of health care work forces, including trainees, have already been identified in this crisis and should be used to provide support at both the residency program and institutional or governmental policies. ${ }^{28} \mathrm{~A}$ recent publication addressed the well-being of trainees during COVID-19 and proposed a Modified Maslow's Framework to tackle the needs of the trainees' educational program, within and outside their institution. ${ }^{29}$ Program leaders can take the opportunity to reflect upon their training programs based on this framework and should improve them based on our data.

Besides taking care of patients, trainees are fulfilling an academic training. The COVID-19 pandemic was perceived as an impediment with negative consequences on clinical and technical end terms by the majority of the participants. This was also the case for administrative end terms with some trainees even fearing to fail to achieve their end terms. Medical training centres should be aware of this and should take preventive measurements to obviate the perceived negative impact.

This study has several limitations. The questionnaire is a self-designed questionnaire based on literature and adapted for the local context and was never formally tested for validity and psychometric characteristics. This questionnaire did not have the intention to identify burnout or other psychological conditions, but rather to collect information on the impact of COVID-19 on paediatric trainees. Another limitation is that this study only represents the Flemish paediatric trainees and our educational setting in Flanders may differ from the educational setting elsewhere which can interfere with future comparisons. Because the questionnaire was anonymised, it was not possible to determine how many trainees answered the questionnaire twice.

In the aftermath of COVID-19, it is not unlikely that the burden on paediatrics might be undervalued when essential policy decisions are being taken. Based on the findings described above and without compromising the appreciation for their respective efforts, one should be cautious not to allocate all possible resources solely towards the specialities that were most prominently on the front lines of COVID-19 patient care (e.g., emergency medicine, critical care, internal medicine). Considering that both budgetary and organizational resources will always be limited in health care systems, the post-pandemic period will inescapably require protracted and dire policy decisions to allow for an optimal recovery from the psychosocial and economic harm done by this virus. This survey might contribute to the plea that paediatricians, and their trainees, should not be forgotten in this aftermath.

\section{Conclusion}

Paediatric trainees were affected by the SARS-CoV-2 pandemic on essential domains of occupational and educational activities and emotional well-being. The results of this cohort study should be considered in policy making and resource distribution in the early aftermath of the pandemic, with the plea that paediatricians and their trainees should not be forgotten when allocating educational and psychosocial support.

\section{Acknowledgements}

The authors would specifically like to acknowledge the exceptional efforts made by many trainees in paediatrics in the past period, especially to those who were willing 
to share their information openly and thus contribute to this analysis and synthesis. Analysing the data and drafting the final manuscript was a collaborative effort in name of the junior association of the Flemish Society for Paediatrics (Jong VVK). There were no sources of financial support.

\section{Disclosure of potential conflicts of interest}

None of the authors declared any conflict of interest. Each author submitted to the corresponding author a separate filled in ICMJE form for disclosure of potential conflicts of interest which the corresponding author keeps.

\section{Ethical Approval}

No ethical approval required.

\section{Data statement}

The data that support the findings of this study are available from the corresponding author, Hoste L., upon reasonable request.

This paper was published in the Belgian Journal of Pediatrics (BJP 2021; 2466-8907). The Journal and authors are pleased that the paper is also published in The Indonesian Journal of Community and Occupational Medicine.

\section{References}

2. Nicola M, Alsafi Z, Sohrabi C, Kerwan A, Al-Jabir A, Iosifidis $\mathrm{C}$, et al. The socio-economic implications of the coronavirus pandemic (COVID-19): A review. Int J Surg. 2020;78:185-93.

3. Mortality Analyses, Johns Hopkins University \& Medicine [updated 30 November 2020], available from: https:// coronavirus.jhu.edu/data/mortality

4. Greenberg N, Docherty M, Gnanapragasam S, Wessely S. Managing mental health challenges faced by healthcare workers during covid-19 pandemic. BMJ. 2020;368:m1211.

5. Breazzano MP, Shen J, Abdelhakim AH, Glass LRD, Horowitz JD, Xie SX, et al. New York City COVID-19 resident physician exposure during exponential phase of pandemic. J Clin Invest. 2020;130:4726-33.

6. Gupta T, NazifTM, Vahl TP, et al. Impact of the COVID-19 pandemic on interventional cardiology fellowship training in the New York metropolitan area: A perspective from the United States epicenter. Catheter Cardiovasc Interv. 2020;1-5. https:// doi.org/10.1002/ccd.28977.

7. Khusid JA, Weinstein CS, Becerra AZ, Kashani M, Robins DJ, Fink LE, et al. Well-being and education of urology residents during the COVID-19 pandemic: Results of an American
National Survey. Int J Clin Pract. 2020;74:e13559.

8. Porpiglia F, Checcucci E, Amparore D, Verri P, Campi R, Claps $\mathrm{F}$, et al. Slowdown of urology residents' learning curve during the COVID-19 emergency. BJU Int. 2020;125:E15-E17.

9. Zingaretti N, Contessi Negrini F, Tel A, Tresoldi MM, Bresadola V, Parodi PC. The Impact of COVID-19 on Plastic Surgery Residency Training. Aesthetic Plast Surg. 2020;44:1381-5.

10. Alhaj AK, Al-Saadi T, Mohammad F, Alabri S. Neurosurgery Residents' Perspective on COVID-19: Knowledge, Readiness, and Impact of this Pandemic. World Neurosurg. 2020;139:e848-e858.

11. Alvin MD, George E, Deng F, Warhadpande S, Lee SI. The Impact of COVID-19 on Radiology Trainees. Radiology. 2020;296:246-8

12. De Luca P, Colacurcio V, De Bonis E, Petrosino M, Bisogno A, Troisi D, et al. Impact of the COVID-19 Pandemic on Otolaryngology Residency: A Real-Life Experience. Ear Nose Throat J. 2020;99:563-4.

13. Mishra D, Nair AG, Gandhi RA, Gogate PJ, Mathur S, Bhushan P, et al. The impact of COVID-19 related lockdown on ophthalmology training programs in India - Outcomes of a survey. Indian J Ophthalmol. 2020;68:999-1004.

14. Anwar A, Seger C, Tollefson A, Diachun CAB, Tanaka P, Umar S. Medical education in the COVID-19 era: Impact on anesthesiology trainees. J Clin Anesth. 2020;66:109949.

15. Caruana EJ, Patel A, Kendall S, Rathinam S. Impact of coronavirus 2019 (COVID-19) on training and well-being in subspecialty surgery: A national survey of cardiothoracic trainees in the United Kingdom. J Thorac Cardiovasc Surg. 2020;160:980-7.

16. Kadhum M, Farrell S, Hussain R, Molodynski A. Mental wellbeing and burnout in surgical trainees: implications for the post-COVID-19 era. Br J Surg. 2020;107:e264.

17. Klasen JM, Vithyapathy A, Zante B, Burm S. "The storm has arrived": the impact of SARS-CoV-2 on medical students. Perspect Med Educ. 2020;9:181-5.

18. Sanghavi PB, Au Yeung K, Sosa CE, Veesenmeyer AF, Limon JA, Vijayan V. Effect of the Coronavirus Disease 2019 (COVID-19) Pandemic on Pediatric Resident Well-Being. J Med Educ Curric Dev. 2020. Doi:10/1177/2382120520947062.

19. Babal JC, Webber SA, Ruedinger E. First, Do No Harm: Lessons Learned From a Storytelling Event for Pediatric Residents During the COVID-19 Pandemic. Acad Pediatr. 2020;20:761-2.

20. Keswani RN, Sethi A, Repici A, Messmann H, Chiu PW. How To Maximize Trainee Education During the Coronavirus Disease-2019 Pandemic: Perspectives From Around the World. Gastroenterology. 2020;159:26-9.

21. García-Lozano JA, Cuellar-Barboza A, Garza-Rodríguez V, Vázquez-Martínez O, Ocampo-Candiani J. Dermatologic surgery training during the COVID-19 era. J Eur Acad Dermatol Venereol. 2020;34:e370-e372.

22. Hall AK, Nousiainen MT, Campisi P, Dagnone JD, Frank JR, Kroeker KI, et al. Training disrupted: Practical tips for 
supporting competency-based medical education during the COVID-19 pandemic. Med Teach. 2020;42:756-61.

23. Ishak WW, Lederer S, Mandili C, Nikravesh R, Seligman L, Vasa M, et al. Burnout during residency training: a literature review. J Grad Med Educ. 2009;1:236-42.

24. Dimitriu MCT, Pantea-Stoian A, Smaranda AC, Nica AA, Carap AC, Constantin VD, et al. Burnout syndrome in Romanian medical residents in time of the COVID-19 pandemic. Med Hypotheses. 2020;144:109972.

25. Harrington RA, Elkind MSV, Benjamin IJ. Protecting Medical Trainees on the COVID-19 Frontlines Saves Us All. Circulation. 2020;141:e775-e777.

26. Shaw SCK. Hopelessness, helplessness and resilience: The importance of safeguarding our trainees' mental wellbeing during the COVID-19 pandemic. Nurse Educ Pract.
2020;44:102780.

27. Zhang Y, Ma ZF. Impact of the COVID-19 Pandemic on Mental Health and Quality of Life among Local Residents in Liaoning Province, China: A Cross-Sectional Study. Int J Environ Res Public Health. 2020;17:E2381.

28. Bloom DE, Cadarette D. Infectious Disease Threats in the Twenty-First Century: Strengthening the Global Response. Front Immunol. 2019;10:549.

29. Ripp J, Peccoralo L, Charney D. Attending to the Emotional Well-Being of the Health Care Workforce in a New York City Health System During the COVID-19 Pandemic. Acad Med. 2020;95:1136-9.

30. Weiss PG, Li ST. Leading Change to Address the Needs and Well-Being of Trainees During the COVID-19 Pandemic. Acad Pediatr. 2020;20:735-41. 